\title{
Endocarditis of the tricuspid valve associated with congenital coronary arteriovenous fistula
}

\author{
Mei Lin Ong
}

\begin{abstract}
A 17 year old girl with a congenital right coronary artery to coronary sinus fistula presented with recurrent septic pulmonary embolism secondary to tricuspid valve endocarditis. The diagnosis was made on the basis of echocardiography and cardiac angiography.
\end{abstract}

(Br Heart F 1993;70:276-278)

Congenital coronary arteriovenous fistula is a rare abnormality that is found in 1 out of 50000 children with congenital heart disease. ${ }^{1}$ It can be difficult to diagnose by conventional echocardiography ${ }^{2}$ but Doppler colour flow mapping may be helpful. ${ }^{34}$ Cardiac angiography is still required for a definitive diagnosis in some cases. Most patients are symptom free and infective endocarditis is rare $(0.001$ per patient-year $) .{ }^{56}$

We report a patient with congenital coronary arteriovenous fistula that presented in an unusual manner-as recurrent septic pulmonary embolism secondary to infective endocarditis.

Medicine University of Malaya, Kuala Lumpur, Malaysia M L Ong

Correspondence to: Dr M L Ong, Departmen of Medicine, University of Malaya, 59100 Kuala Lumpur, Malaysia

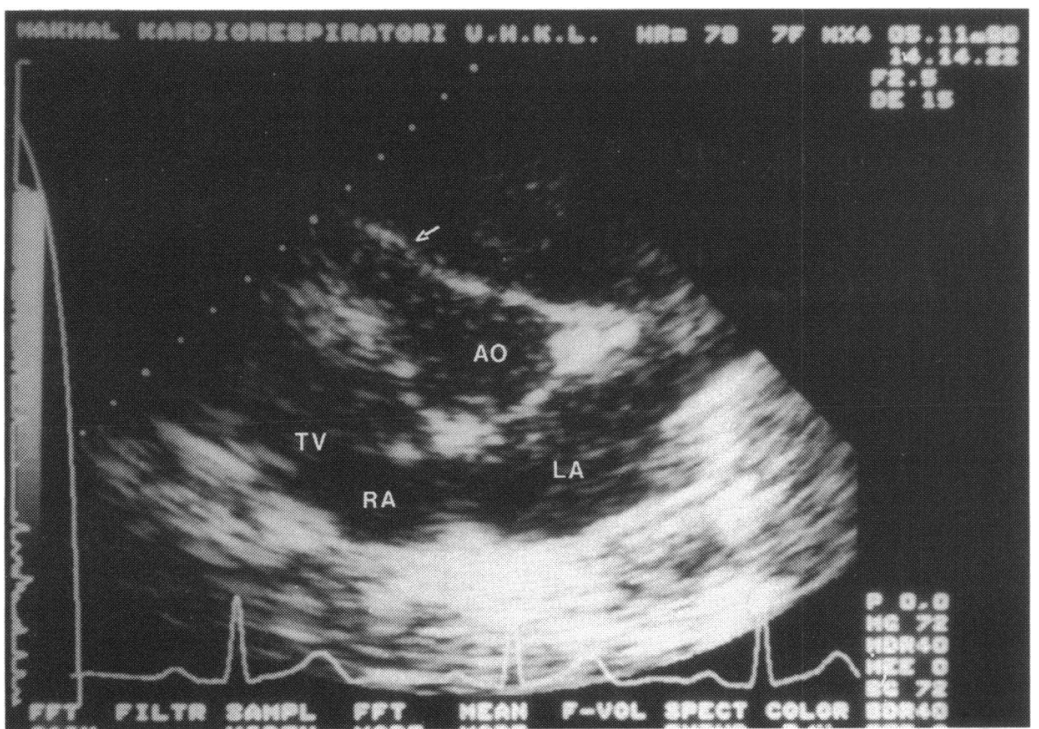

Figure 1 Parasternal short axis echocardiogram showing the proximal portion of the right coronary artery fistula (arrowed). $A O$, aorta; $L A$, left atrium; $R A$, right atrium; $T V$, tricuspid valve. recurrent haemoptysis associated with pleuritic chest pain in the preceding nine months. She had lost $9 \mathrm{~kg}$ and had been amenorrhoeic for four months. She was in New York Heart Association functional class II. There was no history of a cardiac abnormality. She had been treated with several short courses of antibiotics and had a blood transfusion before referral.

On examination she was pale and had a temperature of $38^{\circ} \mathrm{C}$. There was no jaundice, clubbing, or evidence of peripheral embolisation. Her blood pressure was $135 / 70 \mathrm{~mm} \mathrm{Hg}$ and she had a tachycardia of 110 /minute with a bounding pulse. There was no cardiomegaly and heart sounds were normal. A grade $3 / 6$ continuous murmur was heard over the left sternal border. The respiratory system and abdominal examination were normal.

The haemoglobin concentration was $7 \cdot 7$ $\mathrm{g} / \mathrm{dl}$ and the total white cell count was 14 $200 / \mu 1$ with $78 \%$ neutrophils. The erythrocyte sedimentation rate was $68 \mathrm{~mm} / \mathrm{h}$. Biochemical tests were normal and urinalysis showed 80 red blood cells $/ \mu \mathrm{l}$. There was patchy shadowing in the right posterior basal segment on the chest $x$-ray. The electrocardiogram showed an $S$ wave in lead I and a $Q$ wave with $T$ wave inversion in lead III. A standard cross sectional echocardiogram was performed as well as Doppler echocardiogram with colour flow mapping (Toshiba Colour Doppler Ultrasonograph model SH $65 \mathrm{~A})$. The cardiac chambers were normal in size but there was an aneurysmal dilatation of the proximal right coronary artery/sinus of Valsalva (fig 1). The mid and distal right coronary artery was not visualised. The coronary sinus was prominent. There was a large vegetation on the tricuspid valve (fig 2). Doppler examination showed turbulent flow above the aortic valve at the right sinus of Valsalva but no definite fistula draining into a cardiac chamber. Transoesophageal echocardiography was not available at that time.

The provisional diagnoses were ruptured sinus of Valsalva or congenital coronary arteriovenous fistula associated with tricuspid valve endocarditis and recurrent septic pulmonary embolism. Blood cultures subsequently grew Streptococcus viridans.

She was treated with a six week course of antibiotics (crystalline penicillin and gentamicin). Her recovery was uneventful except for a further episode of pulmonary embolism after three weeks' treatment. The diagnosis was based on a history of pleuritic chest pain and haemoptysis associated with hypoxaemia. 


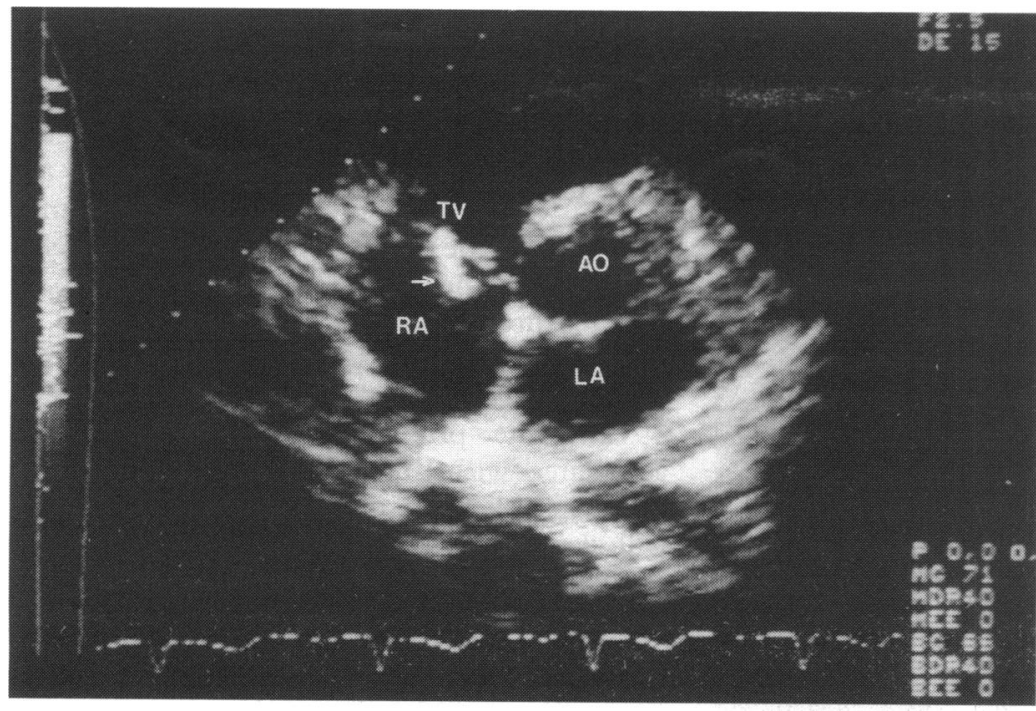

Figure 2 Parasternal short axis echocardiogram showing the vegetation (arrowed) on the tricuspid valve $(T V)$. $A O$, aorta; $L A$, left atrium; $R A$, right atrium.

Figure 3 Supravalvar aortogram in left anterior oblique projection showing the large right coronary artery $(R C A)$ and the fistula $(F)$ ending in the coronary sinus $(S)$. There is a slight opacification of the right atrium $(R A)$ and ventricle $(V)$.

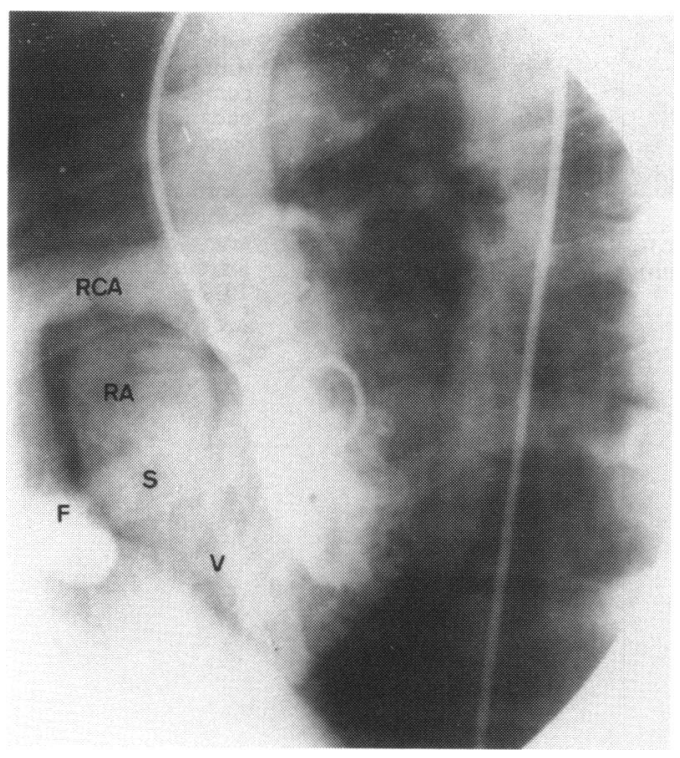

Cardiac catheterisation was performed to obtain an anatomical diagnosis. A supravalvar aortogram (fig 3) showed a grossly dilated right coronary artery with a fistulous communication with the coronary sinus. There was a small left to right shunt with a pulmonary to systemic flow ratio $(\mathrm{Qp} / \mathrm{Qs})$ of 1·4:1.

\section{Discussion}

The presentation of this patient with congenital coronary arteriovenous fistula was unusual.

This girl probably had had infective endocarditis for over a year. Turbulence over the tricuspid valve caused by the increased flow could account for the site of the vegetations on the tricuspid valve. Though infective endocarditis is a known complication of congenital coronary arteriovenous fistula it is rare. ${ }^{56}$ To my knowledge congenital coronary arteriovenous fistula presenting as recurrent pulmonary embolism has not been reported before.

Cross sectional echocardiography with Doppler colour flow mapping was successful in diagnosing congenital coronary arteriovenous fistula ${ }^{346}$ in some patients. However, it has its limitations ${ }^{23}$ and cardiac angiography has often been necessary to establish the diagnosis. In my patient the mid and distal portions of the right coronary artery were not visualised and hence the provisional diagnoses included ruptured sinus of Valsalva aneurysm. This limitation of cross sectional echocardiographic diagnosis has been reported before. ${ }^{23}$ The additional use of Doppler colour flow mapping was not helpful in locating the distal shunt site in this patient, perhaps because the shunt was small. Transoesophageal echocardiography might have been helpful. The diagnosis of congenital coronary arteriovenous fistula was confirmed only by cardiac angiography.

I thank Miss MN Lim and Mr JM Tan for technical assistance.

1 Wenger NK. Rare causes of coronary heart disease. In'Hurst JW, ed. The heart. New York: McGraw Hill, $1978 ; 1348-9$.

2 Miyatake $\mathrm{K}$, Okamoto $\mathrm{M}$, Kinoshita $\mathrm{N}$, Fusejima $\mathrm{K}$, Sakakibara H, Nimura Y. Doppler echocardiographic features of coronary arteriovenous fistula. Br Heart $\mathcal{f}$ 1984;51:508-18.

3 Kimball TR, Daniels SR, Meyer RA, Knilas TK, Plowden JS, Schwartz DC. Colour flow mapping in the diagnosis of coronary artery fistula in the neonate: Benefits and limitations. Am Heart f 1989;117:968-7

$4 \mathrm{Ke}$ WL, Wang NK, Lin YM, Shen CT, Chen CL. Right coronary artery fistula into right atrium: diagnosis by colour Doppler echocardiography. Am Heart $\mathcal{F}$ 1988;116:886-9.

5 Liberthson RR, Sagar K, Berkoben JP, Weintraub RM Levine FH. Congenital coronary arteriovenous fistula. Circulation 1979;59:849-54.

6 Rittenhouse EA, Doty DB, Ehrenhaft JL. Congenital coronary artery-cardiac chamber fistula. Ann Thorac Surg $1975 ; 20: 468-85$.

\section{Comment}

The case report above describes a patient with a coronary artery fistula that was complicated by endocarditis. ${ }^{1}$ Clinical presentation of this congenital anomaly resembles that of aortic regurgitation and when aortic regurgitation has been excluded by echocardiographic and Doppler studies the differential diagnosis that remains is ruptured sinus of Valsalva or a coronary artery fistula communicating with either the left or the right side of the heart. When the coronary sinus is the recipient chamber echocardiography should detect the enlarged coronary sinus, which helps to establish the diagnosis.

Infective endocarditis is an important complication of coronary artery fistula. In 1975 we encountered a patient in whom a coronary artery/coronary sinus fistula had been diagnosed during life, and who subsequently died despite appropriate antibiotic therapy for endocarditis. At necropsy we found that the site of the endocarditis was on a jet lesion on the wall of the coronary sinus opposite the site of entry of the coronary artery and not as 